\title{
Fluidity, anisotropy, and velocity correlations in frictionless, collisional grain flows
}

\author{
Diego Berzi \\ Department of Civil and Environmental Engineering, Politecnico di Milano, 20133 Milano, Italy \\ James T. Jenkins \\ School of Civil and Environmental Engineering, Cornell University, Ithaca, New York 14853, USA
}

(Received 19 September 2017; published 7 September 2018)

\begin{abstract}
We show that the fluidity, made dimensionless by the square root of the granular temperature, measured in numerical simulations of granular shearing flows of frictionless spheres, may be predicted over a wide range of volume fractions using existing kinetic theories. We also show that the departure of these predictions from the measurements in the limit of random close packing is due to the reduction of the dissipation because of velocity correlations and the contribution of the anisotropy of the second moment of the velocity fluctuations to the shear stress. The former causes the granular temperature to increase without bound, the latter ensures that the pressure and shear stress behave in the same way with temperature. This combination of mechanisms may also be relevant to the shearing of emulsions, dense colloids, and non-Brownian suspensions.
\end{abstract}

DOI: 10.1103/PhysRevFluids.3.094303

\section{INTRODUCTION}

Continuum models of dry granular flows rely on constitutive relations for the stresses. In the past decade, inertial granular rheology [1] based on a phenomenological local relation between the ratio of the particle shear stress $s$ to the particle pressure $p$ and the inertial number (the shear rate $\dot{\gamma}$ made dimensionless using the particle pressure, diameter $\sigma$, and mass density $\rho_{p}$ ) that includes an initial yield has become the paradigm for dense granular flows at large solid volume fraction $\nu$.

However, several nonlocal effects cannot be captured by the inertial rheology, including the existence of a minimum height for having steady flows over inclines [2,3] and motion observed in regions in which the stress ratio is less than the yield value [4]. The introduction of the concept of granular fluidity $g$ [5], defined as $\dot{\gamma} p / s$, and a partial differential equation that governs its temporal and spatial evolution in the flow permits these limitations to be overcome [6,7]. Fluidity was also seen to collapse the data for inhomogeneous flows in which the corresponding components of the stress differed [8].

Fluidity was introduced in the context of emulsions [9] and had the clear physical meaning of inverse viscosity; however, the interpretation of the fluidity in granular flows is less apparent and controversial [10]. Recently [8], discrete numerical simulations of homogeneous and inhomogeneous shear flows suggested that the granular fluidity is the product of a function of the solid volume fraction and the ratio of the square root of the granular temperature $T$ to the particle diameter $\sigma$. The granular temperature is a measure of the intensity of the particle agitation that is crucial in kinetic theory of granular gases [11,12]. Two possible explanations for this have been proposed [8]: one, that kinetic theories of granular gases, originally intended for dilute flows, apply also to dense flows; the other, that fluctuation-activated processes similar to those introduced by Eyring [13] are responsible [14]. 
Here we wish to first place the results of the numerical simulations [8] that relate fluidity to the granular temperature in the context of existing kinetic theories over a broad range of volume fractions. We then focus on dense shearing flows for which departures are observed.

\section{THEORY}

Although several kinetic theories of granular gases have been developed over the past 40 years, all predict that the granular pressure and shear stress have the forms

$$
p=\rho_{p} f_{1} T
$$

and

$$
s=\rho_{p} \sigma T^{1 / 2} f_{2} \dot{\gamma},
$$

where $f_{1}$ and $f_{2}$ are known dimensionless functions of $v$, both directly and through the radial distribution function at contact $\chi_{0}$ for a colliding pair of spheres [15], the coefficient of normal restitution $e$ between pairs of colliding spheres, and $\sigma \dot{\gamma} / T^{1 / 2}$, either directly or through dimensionless measures of the second moment of the velocity fluctuations [16]. With Eqs. (1) and (2), the granular fluidity is

$$
g=\frac{\dot{\gamma} p}{s}=\frac{T^{1 / 2}}{\sigma} \frac{f_{1}}{f_{2}}
$$

consistent with findings in numerical simulations [8].

The detailed forms of the coefficients $f_{1}$ and $f_{2}$ depend on the approximations that are employed in deriving the constitutive relations of kinetic theory. If the deviatoric part of the tensor of the second moment of the velocity fluctuations is assumed to be small compared to its isotropic part and only terms proportional to the shear rate are retained in the constitutive relations, the coefficients $f_{1}$ and $f_{2}$ are functions only of $v$ and $e$ (Navier-Stokes order). If, on the other hand, anisotropy in the second moment tensor of the velocity fluctuations is taken to be significant, the constitutive relations also contain terms of higher order in the shear rate through the dependence of $f_{1}$ and $f_{2}$ on $\sigma \dot{\gamma} / T^{1 / 2}$ [16]. Explicit forms of $f_{1}$ and $f_{2}$ for dense flows in the theory of Saha and Alam [16] are provided later.

Figure 1 shows a comparison of the measurements of the dimensionless fluidity $\sigma g / T^{1 / 2}$ obtained in discrete numerical simulations of homogeneous shear flows of rigid [17] and slightly compliant [18,19] frictionless spheres, with a coefficient of restitution equal to 0.7 , and the corresponding ratio $f_{1} / f_{2}$ from the isotropic Navier-Stokes-level kinetic theory of Garzó and Dufty [20] and the anisotropic higher-order kinetic theory of Saha and Alam [16]. The anisotropy is associated with the components of the second moment $\langle\mathbf{C C}\rangle$ of the velocity fluctuations $\mathbf{C}$ with respect to the principal axes in a steady homogeneous shearing flow, in which the $x$ and $y$ axes are in the directions of greatest stretching and compression, respectively, and the $z$ axis is normal to them,

$$
T\left[\begin{array}{ccc}
1+\lambda^{2}-\eta & 0 & 0 \\
0 & 1+\lambda^{2}+\eta & 0 \\
0 & 0 & 1-2 \lambda^{2}
\end{array}\right],
$$

where $T=\left\langle C^{2}\right\rangle / 3, \eta$ is the difference in strength of the velocity fluctuations in the shear plane parallel and perpendicular to the flow, and $\lambda^{2}$ is the difference in strength of the velocity fluctuations in the shear plane and perpendicular to it, both made dimensionless using $T$.

There are two points to be made in regard to this figure. First, the predictions of the kinetic theory of Saha and Alam are in excellent agreement with the numerical simulations for almost the entire range of solid volume fractions, while those of the kinetic theory of Garzó and Dufty are qualitatively similar, but slightly underestimate the measurements. That is, measurements of fluidity 


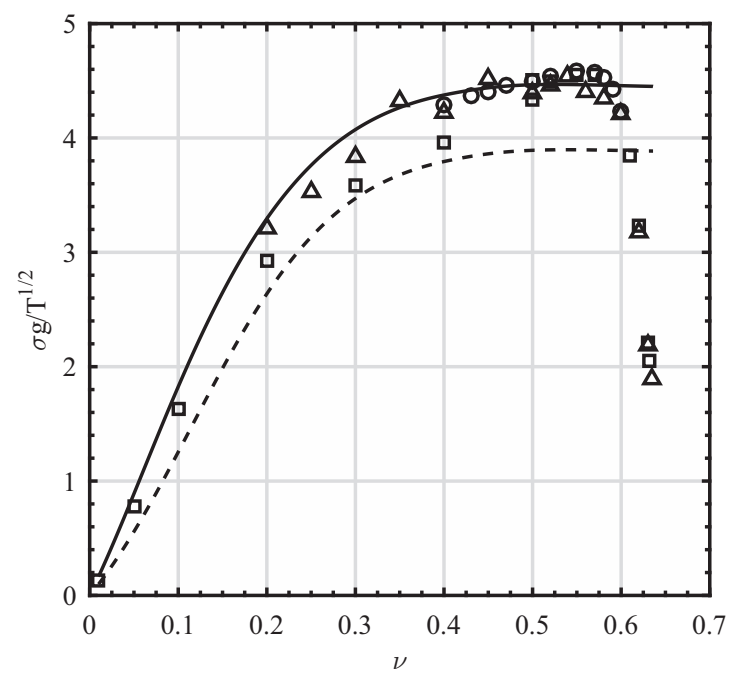

FIG. 1. Dimensionless fluidity as a function of the solid volume fraction in steady, homogeneous shearing flows of frictionless spheres with a coefficient of normal restitution equal to 0.7: measurements in event-driven (circles, [17]) and soft-sphere (squares, [18]; triangles, [19]) molecular dynamics simulations, predictions of kinetic theory at the isotropic Navier-Stokes order (dashed line, [20]), and the anisotropic higher-order kinetic theory (solid line, [16]).

can be predicted by kinetic theories over a wide range of volume fractions. Second, both theories fail in reproducing the drop in the dimensionless fluidity at volume fractions approaching random close packing $(v=0.64)$. A similar drop in the dimensionless fluidity is also observed by Zhang and Kamrin [8] in the numerical simulations of frictional, very inelastic spheres. The remainder of this work will address the reason for the drop in homogeneous flows of frictionless spheres in the context of the kinetic theory.

At volume fractions greater than $0.49, \chi_{0}$ is large compared to $v$ and the contribution of transport to the stresses is less than $10 \%$ that of collisions. In this dense limit, the second-order (Burnett) theory of Saha and Alam involves $R=\sigma \dot{\gamma} / 8 T^{1 / 2}$, a dimensionless measure of the shear rate, and $\eta$. To second order in $R$ and $\eta$, the coefficients $f_{1}$ and $f_{2}$ are

$$
f_{1}=2(1+e) v^{2} \chi_{0}\left(1+\frac{224}{105} R^{2}+\frac{112}{105 \pi^{1 / 2}} \eta R\right)
$$

and

$$
f_{2}=\frac{4(1+e)}{5 \pi^{1 / 2}} v^{2} \chi_{0}\left(1+\pi^{1 / 2} \frac{\eta}{8 R}\right)
$$

The Garzó-Dufty theory involves only the first of the terms in large parentheses in Eq. (5) and a slightly different dependence on $e$ in Eq. (6). The quadratic term in Eq. (5) is a Burnett-order nonlinearity; the terms that involve $\eta$ in Eqs. (5) and (6) permit the isotropic and deviatoric parts of the flow to contribute, respectively, to the deviatoric and isotropic parts of the stress. Because $\lambda^{2}$ is second order in $R$ and $\eta$ [16], its contributions to the stresses is greater than second order and can be ignored.

Using Eqs. (5) and (6) in Eq. (3) gives

$$
\frac{\sigma g}{T^{1 / 2}}=\frac{4\left(105 \pi^{1 / 2}+224 \pi^{1 / 2} R^{2}+112 \eta R\right)}{21\left(8+\pi^{1 / 2} \eta / R\right)} .
$$


In steady homogeneous shearing, $\eta$ and $R$ are determined by the in-plane deviatoric component and the trace of the balance of the second moment of the velocity fluctuations. The balance equation relates the collisional flux $\boldsymbol{\Theta}$ and collisional production $\boldsymbol{\Gamma}$ of second moment.

The equation for the in-plane deviatoric component of the balance is

$$
\dot{\gamma}\left(\Theta_{x x}+\Theta_{y y}\right)=\Gamma_{x x}-\Gamma_{y y},
$$

where, from Saha and Alam [16], at second order in $R$ and $\eta$,

$$
\Theta_{x x}+\Theta_{y y}=\frac{4(1+e) \rho_{p} v^{2} \chi_{0} T}{\pi^{1 / 2}}
$$

and

$$
\Gamma_{x x}-\Gamma_{y y}=\frac{1008(1+e) \rho_{p} v^{2} \chi_{0} T^{3 / 2}}{105 \sigma \pi^{1 / 2}}\left[(1-e)\left(2 \pi^{1 / 2} R+\eta\right)+2 \eta+2 R \pi^{1 / 2}\right] .
$$

Therefore,

$$
\frac{\dot{\gamma} \sigma}{T^{1 / 2}} 5 \pi^{1 / 2}=12\left[(1-e)\left(2 \pi^{1 / 2} R+\eta\right)+2 \eta+2 R \pi^{1 / 2}\right]
$$

or

$$
10 \pi^{1 / 2} R=6 \pi^{1 / 2}[(1-e)+1] R+3[(1-e)+2] \eta .
$$

Thus,

$$
\eta=\frac{2 \pi^{1 / 2}}{3(3-e)}[5-3(2-e)] R .
$$

The equation for the trace of the balance is

$$
\dot{\gamma}\left(\Theta_{x x}-\Theta_{y y}\right)=A_{x x}+A_{y y}+A_{z z},
$$

where, from Saha and Alam [16], at second order,

$$
\Theta_{x x}-\Theta_{y y}=-\frac{8(1+e) \rho_{p} v^{2} \chi_{0} T}{35 \pi^{1 / 2}}\left(56 R+7 \pi^{1 / 2} \eta\right)
$$

and

$$
A_{x x}+A_{y y}+A_{z z}=-\frac{4\left(1-e^{2}\right) \rho_{p} \nu^{2} \chi_{0} T^{3 / 2}}{35 \sigma \pi^{1 / 2}}\left(210+672 R^{2}+21 \eta^{2}+2 \pi^{1 / 2} \eta R\right) .
$$

Thus, Eq. (14) is

$$
16 R\left(\pi^{1 / 2} \eta+8 R\right)=3(1-e)\left(10+32 R^{2}+\eta^{2}+8 \pi^{1 / 2} \eta R\right),
$$

where the Garzó-Dufty [20] theory involves only the first of the last four terms on the right, with a slightly different dependence on $e$.

Equations (13) and (17) determine $\eta$ and $R$. Consequently, in both theories, $\eta$ is proportional to $R$ [Eq. (13)], $R$ depends only on $e$ [Eq. (17)], and the fluidity is independent of $v$ [Eq. (7)]. This explains the plateau in the predictions as random close packing is approached in Fig. 1.

The question is then what induces the drop in the dimensionless fluidity of Fig. 1. It has been suggested [8] that in an Eyring-like [13] fluctuation-activated model of dense flows, the drop has to do with the decrease of the fraction of sites able to shear with increases in the solid volume fraction. This description might apply to flows of sufficiently rigid particles at solid volume fractions beyond a value at which random aggregates of spheres develop a rate-independent component to the stresses $[21,22]$. Another way to obtain the drop is to employ different exponents for the singularities in the volume fraction dependence of the pressure, shear stress, and temperature. Vescovi and Luding [19] employ functions obtained through fits to numerical simulations to do this. Here we provide an alternative explanation based on kinetic theory that highlights the mechanisms that are responsible 
for the observed behavior. It is based on the existence of velocity correlations, their influence on the collisional dissipation of the flow, and the presence of anisotropy in the second moment of the velocity fluctuations.

Extended kinetic theory [23-25] takes the correlations into account by introducing a phenomenological correlation length $L$ in the collisional rate of dissipation. Its expression, based on a heuristic balance between correlation induced by shear and randomization induced by collisions [24] and, then a fit to numerical simulations, based on the Garzo-Dufty theory [26,27], is

$$
\frac{L}{\sigma}=\left[\frac{2 J}{15\left(1-e^{2}\right)}\right]^{1 / 2}\left[\frac{26(1-e)}{15}\left(\frac{v-0.49}{0.64-v}\right)+1\right]^{3 / 2} \frac{\sigma|\dot{\gamma}|}{T^{1 / 2}} \equiv 8 f_{0}|R|,
$$

where $J=(1+e) / 2+\pi(1+e)^{2}(3 e-1) /\left[96-24(1-e)^{2}-20\left(1-e^{2}\right)\right]$.

Here we apply this idea to the second-order theory by incorporating the correlation length into all of the components of the collisional production of second moment. In this case, the right-hand sides of both Eqs. (12) and (17) are divided by $L / \sigma=8 f_{0}|R|$ and become

$$
80 \pi^{1 / 2} f_{0}|R| R=6 \pi^{1 / 2}(2-e) R+3(3-e) \eta
$$

and

$$
128 f_{0}|R| R\left(\pi^{1 / 2} \eta+8 R\right)=3(1-e)\left(10+32 R^{2}+\eta^{2}+8 \pi^{1 / 2} \eta R\right) .
$$

The function $f_{0}$, which is singular at random close packing, then influences the determination of $\eta$ in terms of $R$ :

$$
\eta=\frac{2 \pi^{1 / 2}}{3(3-e)}\left[40 f_{0}|R|-3(2-e)\right] R .
$$

When Eq. (21) is used in the energy balance (20), the terms proportional to $f_{0}^{2} R^{4}$ dominate and, at lowest order,

$$
|R|=\frac{1}{f_{0}^{1 / 2}}\left\{\frac{9(3-e)^{2}(1-e)}{8 \pi[128(3-e)-80(1-e)]}\right\}^{1 / 4} .
$$

Then, with Eqs. (21) and (22),

$$
\frac{\eta}{R}=\frac{80 \pi^{1 / 2}}{3(3-e)}\left\{\frac{9(3-e)^{2}(1-e)}{8 \pi[128(3-e)-80(1-e)]}\right\}^{1 / 4} f_{0}^{1 / 2} .
$$

As random close packing is approached, Eqs. (21) and (22) show that $\eta$ goes to a constant and $R$ goes to zero. That is, as a consequence of the increase of velocity correlations, dissipation is diminished and, in the limit, the temperature increases without bound. Thus, in Eq. (7) the term proportional to $\eta / R$ dominates and, in the limit, the dimensionless fluidity goes to zero.

Alternatively, from Eq. (3), the dimensionless fluidity may be written as

$$
\frac{\sigma g}{T^{1 / 2}}=8 \frac{p}{s} R
$$

where

$$
\frac{p}{s}=\frac{f_{1}}{f_{2}} \frac{1}{8 R}
$$

As random close packing is approached, the stress ratio becomes proportional to $\eta$ and therefore a constant. That is, because of the anisotropy in the second moment, the pressure and the shear stress behave in the same way with the temperature and the dimensionless fluidity in Eq. (24) is proportional to $R$.

Figure 2 shows a comparison between the predictions of Eq. (7) and the dimensionless fluidity measured in numerical simulations of steady, homogeneous shearing flows of frictionless spheres 


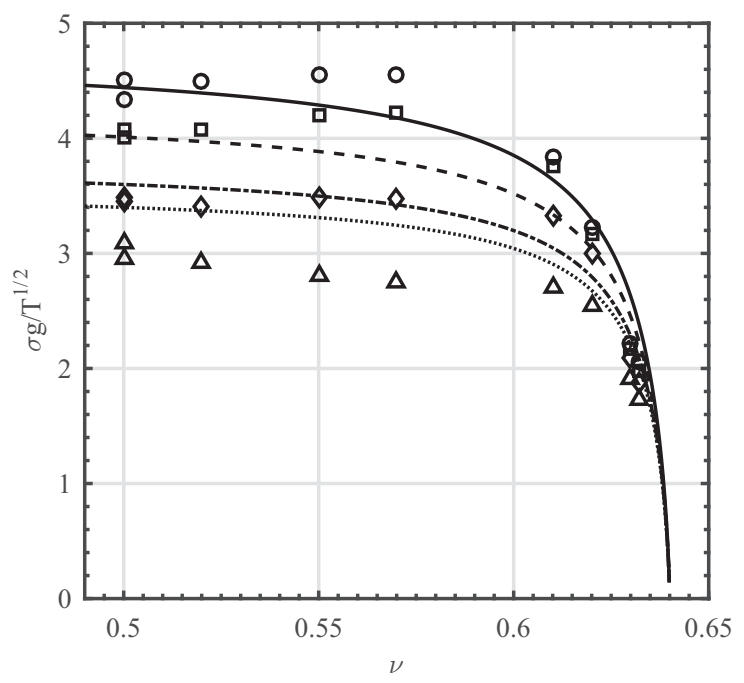

FIG. 2. Measured (symbols, [17,18]) and predicted [lines, Eqs. (15) and (16)] dimensionless fluidity as a function of the solid volume fraction in steady, homogeneous shearing flows of frictionless spheres with coefficient of collisional restitution equal to 0.7 (circles and solid line), 0.8 (squares and dashed line), 0.9 (diamonds and dot-dashed line), and 0.95 (triangles and dotted line).

at different values of the coefficient of restitution. Given the number of approximations made in the course of the present analysis, the agreement seems satisfactory.

\section{CONCLUSION}

We have shown that the granular fluidity, made dimensionless with the square root of the granular temperature, is well predicted by kinetic theory based on the assumption of molecular chaos over a wide range of volume fractions. We have also shown that the departures in these predictions from the data at volume fractions approaching 0.64 can be understood as being due to the reduction of the dissipation due to the velocity correlations and the contribution of the anisotropy of the second moment to the shear stress. The former causes the temperature to increase without bound; the latter ensures that the pressure and shear stress behave in the same way with temperature, in contrast, for example, to the linear theory of Garzó and Dufty [20]. This combination of mechanisms can also influence aspects of the material response other than the fluidity, such as the viscosity and the differences in normal stresses in simple shearing. The determination of how they are influenced is a natural extension of the present work. Such consideration is likely to have relevance in the shearing of dense colloids [28], and non-Brownian suspensions [29]. Finally, it remains to understand how to extend the present analysis to frictional particles and inhomogeneous flows [8].

[1] GDR-MiDi, On dense granular flows, Eur. Phys. J. E 14, 341 (2004).

[2] O. Pouliquen, Scaling laws in granular flows down rough inclined planes, Phys. Fluids 11, 542 (1999).

[3] L. E. Silbert, D. Ertas, G. S. Grest, T. C. Halsey, D. Levine, and S. J. Plimpton, Granular flow down an inclined plane: Bagnold scaling and rheology, Phys. Rev. E 64, 051302 (2001).

[4] G. Koval, J.-N. Roux, A. Corfdir, and F. Chevoir, Annular shear of cohesionless granular materials: From the inertial to quasistatic regime, Phys. Rev. E 79, 021306 (2009). 
[5] K. Kamrin and G. Koval, Nonlocal Constitutive Relation for Steady Granular Flow, Phys. Rev. Lett. 108, 178301 (2012).

[6] D. L. Henann and K. Kamrin, A predictive, size-dependent continuum model for dense granular flows, Proc. Natl. Acad. Sci. USA 110, 6730 (2013).

[7] K. Kamrin and D. L. Henann, Nonlocal modeling of granular flows down inclines, Soft Matter 11, 179 (2015).

[8] Q. Zhang and K. Kamrin, Microscopic Description of the Granular Fluidity Field in Nonlocal Flow Modeling, Phys. Rev. Lett. 118, 058001 (2017).

[9] J. Goyon, A. Colin, G. Ovarlez, A. Ajdari, and L. Bocquet, Spatial cooperativity in soft glassy flows, Nature (London) 454, 84 (2008).

[10] M. Bouzid, A. Izzet, M. Trulsson, E. Clément, P. Claudin, and B. Andreotti, Non-local rheology in dense granular flows, Eur. Phys. J. E 38, 125 (2015).

[11] J. T. Jenkins and S. B. Savage, A theory for the rapid flow of identical, smooth, nearly elastic, spherical particles, J. Fluid. Mech. 130, 187 (1983).

[12] I. Goldhirsch, Rapid granular flows, Annu. Rev. Fluid Mech. 35, 267 (2003).

[13] H. Eyring, The activated complex in chemical reactions, J. Chem. Phys. 3, 107 (1935).

[14] O. Pouliquen and Y. Forterre, A non-local rheology for dense granular flows, Philos. Trans. R. Soc. A 367, 5091 (2009).

[15] S. Torquato, Nearest-neighbor statistics for packing of hard spheres and disks, Phys. Rev. E 51, 3170 (1995).

[16] S. Saha and M. Alam, Normal stress differences, their origin and constitutive relations for a sheared granular fluid, J. Fluid Mech. 795, 549 (2016).

[17] N. Mitarai and H. Nakanishi, Velocity correlations in the dense granular shear flows: Effects on energy dissipation and normal stress, Phys. Rev. E 75, 031305 (2007).

[18] S. Chialvo and S. Sundaresan, A modified kinetic theory for frictional granular flows in dense and dilute regimes, Phys. Fluids 25, 070603 (2013).

[19] D. Vescovi and S. Luding, Merging fluid and solid granular behavior, Soft Matter 12, 8616 (2016).

[20] V. Garzó and J. W. Dufty, Dense fluid transport for inelastic hard spheres, Phys. Rev. E 59, 5895 (1999).

[21] S. Chialvo, J. Sun, and S. Sundaresan, Bridging the rheology of granular flows in three regimes, Phys. Rev. E 85, 021305 (2012).

[22] D. Berzi and D. Vescovi, Different singularities in the functions of extended kinetic theory at the origin of the yield stress in granular flows, Phys. Fluids 27, 013302 (2015).

[23] J. T. Jenkins, Dense shearing flows of inelastic disks, Phys. Fluids 18, 103307 (2006).

[24] J. T. Jenkins, Dense inclined flows of inelastic spheres, Granular Matter 10, 47 (2007).

[25] J. T. Jenkins and D. Berzi, Dense inclined flows of inelastic spheres: Tests of an extension of kinetic theory, Granular Matter 12, 151 (2010).

[26] D. Berzi, Extended kinetic theory applied to dense, granular, simple shear flows, Acta Mech. 225, 2191 (2014).

[27] D. Berzi and J. T. Jenkins, Steady shearing flows of deformable, inelastic spheres, Soft Matter 11, 4799 (2015).

[28] T. N. Phung, J. F. Brady, and G. Bossis, Stokesian dynamics simulation of Brownian suspensions, J. Fluid Mech. 313, 181 (1996).

[29] C. Ness and J. Sun, Flow regime transitions in dense non-Brownian suspensions: Rheology, microstructural characterization, and constitutive modeling, Phys. Rev. E 91, 012201 (2015). 